\title{
X Congreso Ilbérico de Agroingeniería

\section{Integração da condutividade eléctrica do solo e de índices obtidos por imagens de satélite para gestão diferenciada da fertilização em pastagens}

\author{
J. Serrano1, José Marques da Silva², Shakib Shahidian ${ }^{3}$, Christiane Santos $^{4}$, Mário de \\ Carvalho 5
}

1 ICAAM, Departamento de Engenharia Rural, Escola de Ciências e Tecnologia, Universidade de Évora, P.O. Box 94, Évora 7002-554, Portugal; jmrs@uevora.pt

2 ICAAM, Departamento de Engenharia Rural, Escola de Ciências e Tecnologia, Universidade de Évora, P.O. Box 94, Évora 7002-554, Portugal; jmsilva@uevora.pt

3 ICAAM, Departamento de Engenharia Rural, Escola de Ciências e Tecnologia, Universidade de Évora, P.O. Box 94, Évora 7002-554, Portugal; shakib@uevora.pt

4 ICAAM, Universidade de Évora, P.O. Box 94, Évora 7002-554, Portugal, kris_11crsmulek@hotmail.com

5 ICAAM, Departamento de Fitotecnia, Escola de Ciências e Tecnologia, Universidade de Évora, P.O. Box 94, Évora 7002-554, Portugal; mjc@uevora.pt

\begin{abstract}
Resumo: As pastagens de sequeiro no Alentejo, região situada no Sul de Portugal, ocupam normalmente solos que apresentam reduzida fertilidade mas, simultaneamente, grande variabilidade espacial. A aplicação racional de fertilizantes pressupõe o conhecimento da variabilidade espacial do solo e da resposta da cultura, o que exige que tecnologias expeditas possam identificar "zonas homogéneas de gestão" (ZHG). Neste trabalho monitorizou-se, uma parcela de cerca de 25 ha de pastagem sob montado de azinho. Foram realizados dois levantamentos da condutividade eléctrica aparente do solo ( $\mathrm{CE}_{\mathrm{a}}$ ) com um sensor de contacto Veris 2000 XA (em Novembro de 2017 e Outubro de 2018) e foram estabelecidos 24 pontos de amostragem $(30 \mathrm{~m} \times 30 \mathrm{~m})$ em zonas sem árvores para permitir as leituras dos registos históricos dos índices NDVI e NDWI obtidos a partir das imagens de satélite (Sentinel-2) no período de Inverno e Primavera de 2017 e de 2018. Os resultados obtidos ao nível da variabilidade espacial e da estabilidade temporal dos parâmetros medidos permitiram definir ZHG, as quais constituem a base para a elaboração de mapas de prescrição diferenciada de adubos com tecnologia de aplicação variável, tendo em conta a variabilidade das características do solo e do desenvolvimento da cultura e contribuindo para a sustentabilidade deste ecossistema.
\end{abstract}

Palavras-chave: variabilidade espacial, CEa, NDVI, montado, tomada de decisão

\section{Introdução}

As pastagens de sequeiro no Alentejo, região situada no Sul de Portugal, constituem a base da alimentação dos animais em sistemas de produção extensivos. Ocupam normalmente solos que apresentam reduzida fertilidade mas, simultaneamente, grande variabilidade espacial. Esta resulta da conjugação de factores como: relevo ondulado, variabilidade intrínseca ao nível da rocha-mãe originária, existência de afloramentos rochosos, influência das árvores ou o efeito do pastoreio animal [1]. A variabilidade do substrato solo reflecte-se no heterogéneo desenvolvimento da pastagem [2]. A utilização de pastagens biodiversas (que incluem leguminosas, potencialmente fixadoras do azoto atmosférico) e o predomínio nesta região da rocha-mãe originária rica em potássio, determinam como prática comum em pastagens a 


\section{CONGRESO IBÉRICO DE AGROINGENIERÍA \\ X CONGRESSO IBÉRICO DE AGROENGENHARIA \\ 3 - 6 septiembre 2019, Huesca - España}

aplicação anual e homogénea de adubos fosfatados no final do Outono [3]. A primeira etapa para implementação de estratégias de gestão diferenciada dos factores de produção pressupõe determinar os padrões espaciais das principais propriedades do solo e do vigor da vegetação, o que requer o conhecimento da variabilidade do solo e da resposta da cultura, para além da utilização de tecnologias de aplicação variável (VRT) para fechar o ciclo. Estes distribuidores VRT são comuns, especialmente entre os prestadores de serviços, mas exigem que tecnologias expeditas, numa fase prévia, identifiquem zonas com características (do solo e do desenvolvimento da cultura) semelhantes, conhecidas como "zonas homogéneas de gestão (ZHG)".

A utilização da condutividade eléctrica aparente do solo $\left(\mathrm{CE}_{\mathrm{a}}\right)$, por integrar as principais propriedades que afectam a produtividade das culturas (nomeadamente, a textura, a matéria orgânica e a capacidade de troca catiónica do solo), constitui uma das mais apropriadas variáveis do solo para caracterizar zonas de gestão de uma parcela, definir a amostragem inteligente e estabelecer mapas de prescrição da fertilização ou da correcção do solo [1]. Vários estudos demonstraram o interesse prático da monitorização da CEa para estabelecimento de ZHG [4-6], quer pela utilização de sensores de indução electromagnética (como o EM38 ou o DUALEM), quer pela utilização de sensores de contacto (como é o exemplo da tecnologia Véris). Apesar destas tecnologias darem uma resposta expedita, têm um custo por hectare, variável consoante a área a levantar, que pode não ser compatível com a reduzida margem de lucro associada à produção animal extensiva.

Nos últimos anos, o acesso gratuito a imagens de satélite e à obtenção de índices vários, entre eles o NDVI ("normalized difference vegetation índex") e o NDWI ("normalized difference water índex"), proporciona informação com uma resolução espacial muito interessante (de " $10 \mathrm{~m} \times 10 \mathrm{~m}$ " no NDVI e de " $20 \mathrm{~m} \times 20 \mathrm{~m}$ " no NDWI) e com uma resolução temporal de apenas 5 dias.

O NDVI, baseado na medição da radiação reflectida pelas plantas em dois comprimentos de onda: o vermelho ("RED") e o infravermelho próximo ("NIR"), é o índice de vegetação mais utilizado para definição de ZHG [7,8]. Altos valores de NDVI reflectem maior vigor e capacidade fotossintética da vegetação, enquanto valores baixos de NDVI podem reflectir situações de stress das plantas [9,10].

Por outro lado, o NDWI, obtido a partir das bandas "B8A" (865 nm) e "B11" (1610 nm) do espectro do Sentinel-2 foi proposto para descrever o estado da vegetação em termos de teor em água [11,12] ou para descrever o teor de humidade superficial do solo [13]. Valores mais elevados de NDWI indicam maior humidade no solo e nas plantas, enquanto valores mais baixos e até negativos são comuns nas fases finais do ciclo vegetativo das plantas e em solos relativamente secos.

Neste trabalho procura-se demonstrar o interesse de associar o levantamento expedito da $\mathrm{CE}_{a}$ do solo à monitorização de séries temporais de índices obtidos a partir de imagens de satélite com o objectivo de definir e validar ZHG. São utilizadas técnicas para avaliar a variabilidade espacial e a estabilidade temporal dos parâmetros medidos, aspectos fundamentais no apoio à tomada de decisão no que confere à elaboração de mapas de prescrição diferenciada de adubo.

\section{Materiais e métodos}

\subsection{Parcela experimental}

Neste trabalho monitorizou-se uma parcela de cerca de 25 ha de pastagem sob montado de azinho situada na Herdade da Mitra, da Universidade de Évora (coordenadas $38^{\circ} 32,1^{\prime} \mathrm{N}$; $\left.7^{\circ} 59,8^{\prime} \mathrm{W}\right)$. O solo é classificado como Cambissolo derivado de granito [14]. Estes solos são 


\section{CONGRESO IBÉRICO DE AGROINGENIERÍA \\ X CONGRESSO IBÉRICO DE AGROENGENHARIA \\ 3 - 6 septiembre 2019, Huesca - España}

pouco férteis, normalmente ácidos e principalmente usados em sistemas mistos agro-silvopastoris.

Foram estabelecidos 24 pontos de amostragem geo-referenciados (cada um com uma área de $900 \mathrm{~m}^{2}$, correspondente a um "pixel" do "Sentinel-2" de "30m×30m"). Estes foram seleccionados em zonas sem árvores para permitir as leituras dos índices obtidos a partir das imagens de satélite sem interferência da vegetação arbórea.

\subsection{Levantamento altimétrico}

Em Novembro de 2017 foi realizado um levantamento topográfico da área de ensaio utilizando um GPS-RTK (Trimble RTK/ PP - 4700 GPS, Trimble Navigation Limited, USA). O mapa altimétrico da parcela experimental foi criado a partir de interpolação linear em ArcGIS 9.3 com uma grelha de $1 \mathrm{~m}$ de resolução.

\subsection{Levantamento da condutividade eléctrica aparente do solo}

Foram realizados dois levantamentos da condutividade eléctrica aparente do solo com um sensor Veris 2000 XA (em Novembro de 2017 e em Outubro de 2018) na camada de solo de 0$0,30 \mathrm{~m}$. O sensor foi puxado por um tractor agrícola, tendo realizado passagens sucessivas espaçadas $10 \mathrm{~m}$ entre si. O espaçamento entre passagens foi garantido por um sistema GPS de apoio à condução do tractor. Em cada um dos levantamentos da $\mathrm{CEa}$ foram recolhidas 12 amostras de solo (em metade dos pontos de amostragem) para determinação da humidade volumétrica na camada superficial $(0-0,30 \mathrm{~m})$ de acordo com procedimento standard.

\subsection{Determinação das características do solo}

Em Novembro de 2017 foram recolhidas amostras de solo nos 24 pontos de amostragem. A recolha foi realizada manualmente com uma sonda "meia-cana" e um maço na camada de solo 0-0,30 m. As amostras compósitas (resultantes de 5 sub-amostras recolhidas na área do pixel), devidamente codificadas, foram transportadas para o Laboratório de Química Agrícola da Universidade de Évora onde se procedeu à determinação da textura, dos teores de matéria orgânica, do $\mathrm{pH}$, do fósforo, do potássio e da capacidade de troca catiónica de acordo com procedimentos standard.

\subsection{Determinação de indices obtidos a partir das imagens de satélite}

Através da plataforma electrónica "http://agromap.agroinsider360.com" da empresa "AgroInsider" (uma "spin-off" da Universidade de Évora) foram capturados os registos históricos do NDVI e do NDWI no período de Inverno e Primavera de 2017 e de 2018 nos 24 pontos de amostragem (pixeis do "Sentinel-2"). Foram eliminados os registos obtidos em dias com nebulosidade.

\subsection{Tratamento dos dados}

A análise estatística dos resultados incluiu a análise descritiva com o cálculo da média, do desvio padrão (DP), do coeficiente de variação $(\mathrm{CV})$ e do intervalo de variação de cada conjunto de dados.

A estabilidade temporal da $\mathrm{CE}_{\mathrm{a}}$, do NDVI e do NDWI foi determinada calculando o CV de cada ponto de amostragem entre os dois momentos de avaliação (2017 e 2018), método utilizado previamente por outros autores [15-17]. O CV médio para o período de ensaio (2 anos) e para o conjunto de todos os pontos de amostragem foi calculado com o objectivo de evidenciar a magnitude relativa da variação temporal dos parâmetros em análise. A conjugação da análise da variabilidade espacial (com base no valor relativo; identifica se o valor está acima ou abaixo 


\section{CONGRESO IBÉRICO DE AGROINGENIERÍA \\ X CONGRESSO IBÉRICO DE AGROENGENHARIA \\ 3 - 6 septiembre 2019, Huesca - España}

da média) com a estabilidade temporal (tendo em conta o CV) permitiu a elaboração de mapas de classes de gestão. Foram estabelecidas 5 classes [16]: classe 1- valor superior à média do parâmetro na parcela e estável $(\mathrm{CV}<15 \%)$; classe 2- valor superior à média do parâmetro na parcela e moderadamente estável $(15 \%<\mathrm{CV}<25 \%)$; classe 3- valor inferior à média do parâmetro na parcela e estável $(\mathrm{CV}<15 \%) 1$; classe 4- valor inferior à média do parâmetro na parcela e moderadamente estável $(15 \%<C V<25 \%)$; e classe 5 - instável $(C V>25 \%)$.

Os mapas foram elaborados com o módulo "ArcMap"/Spatial Analyst" do programa ARCGIS 9.3 [18], tendo sido utilizado como interpolador o "inverso do quadrado da distância" ("IDW, Inverse Distance Weighting").

\subsection{Correlação entre parâmetros}

A análise de correlação entre parâmetros foi realizada com o pacote de estatística do "20.0 SPSS package for Windows" (SPSS Inc., Chicago, IL, USA) com um nível de significância de $95 \%(\mathrm{p}<0,05)$.

\section{Resultados e discussão}

A Figura 1 ilustra, à esquerda (a), os limites da parcela e a localização dos 24 pontos de amostragem, e à direita (b), o mapa altimétrico. É evidente o relevo ondulado característico da região, o qual tem influência na dinâmica dos nutrientes no solo [17].

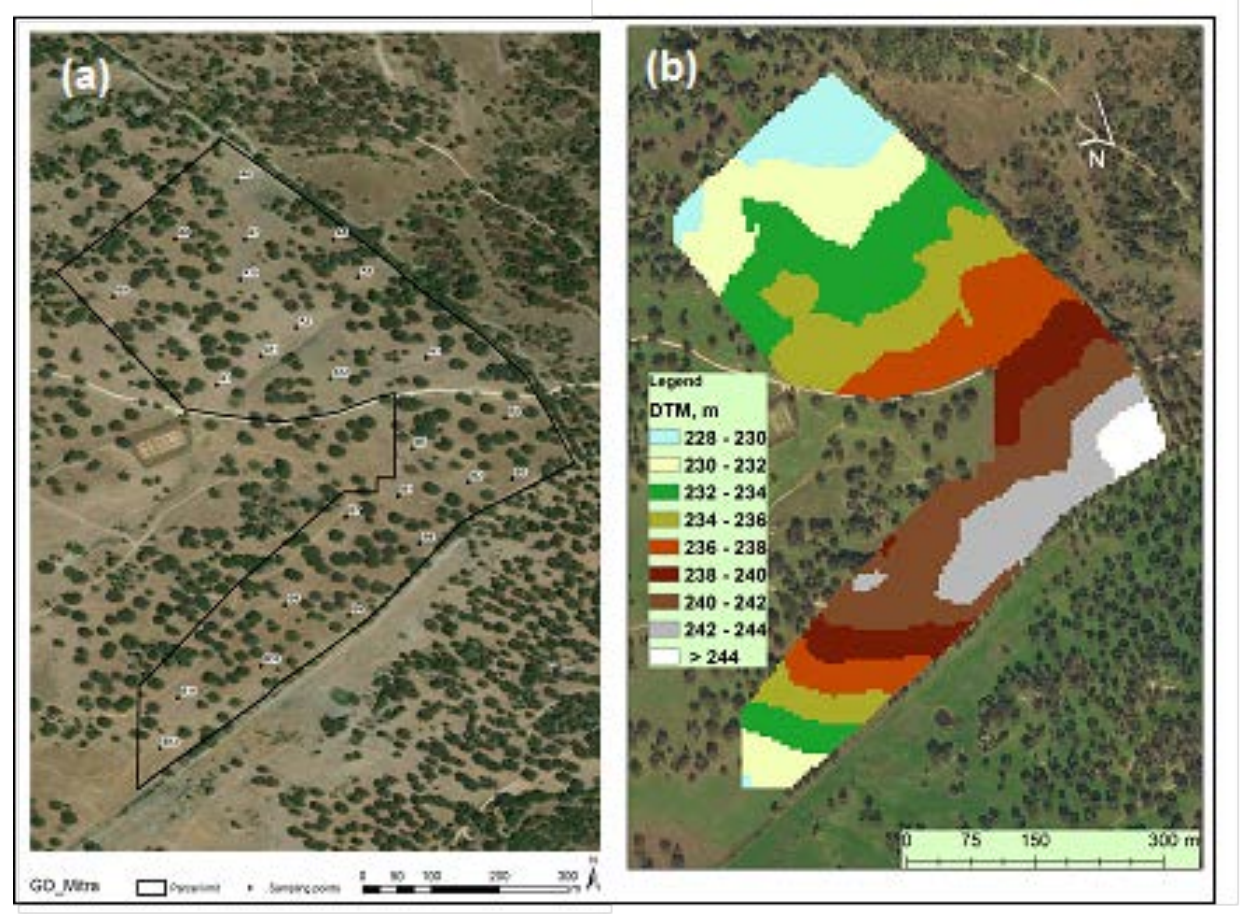

Figura 1. À esquerda limite exterior da parcela de ensaio e localização aproximada dos 24 pontos de amostragem (a); à direita mapa altimétrico (b) da parcela experimental.

A Tabela 1 resume as características da camada superficial do solo $(0-0,30 \mathrm{~m})$ na parcela do campo experimental em Novembro de 2017. Os resultados confirmam as limitações destes tipos de solos, com textura franco-arenosa (teores de argila da ordem dos $10 \%$ ), ácidos (pH da ordem dos 5,5), pobres em fósforo, com fraca capacidade de troca catiónica e baixos teores de matéria orgânica (1,5\% em média). 
X CONGRESO IBÉRICO DE AGROINGENIERÍA

X CONGRESSO IBÉRICO DE AGROENGENHARIA

3 - 6 septiembre 2019, Huesca - España

Tabela 1. Características do solo na camada superficial $(0-0,30 \mathrm{~m})$ da parcela experimental (Novembro de 2017).

\begin{tabular}{cccc}
\hline Parâmetros do solo & Média \pm DP & CV, \% & Intervalo de variação \\
\hline Areia total, \% & $78,4 \pm 4,0$ & 5,0 & {$[71,5-84,6]$} \\
Limo, \% & $11,2 \pm 2,2$ & 19,9 & {$[7,4-15,3]$} \\
Argila, \% & $10,4 \pm 1,8$ & 17,3 & {$[7,2-13,9]$} \\
P2O5, mg/kg & $32,6 \pm 21,5$ & 65,8 & {$[7,8-81,0]$} \\
K2O, mg/kg & $94,0 \pm 72,1$ & 76,7 & {$[18,0-380,0]$} \\
M.O., \% & $1,5 \pm 0,3$ & 21,5 & {$[0,9-2,1]$} \\
pH & $5,5 \pm 0,2$ & 4,4 & {$[5,0-5,8]$} \\
CTC, meq/100g & $10,8 \pm 2,8$ & 26,4 & {$[5,2-17,9]$} \\
\hline
\end{tabular}

DP- Desvio padrão; CV- Coeficiente de variação; M.O.- Matéria orgânica; CTC- Capacidade de troca catiónica;

A Figura 2 ilustra os mapas da CEa na parcela experimental em Novembro de 2017 (a) e em Outubro de 2018 (b).

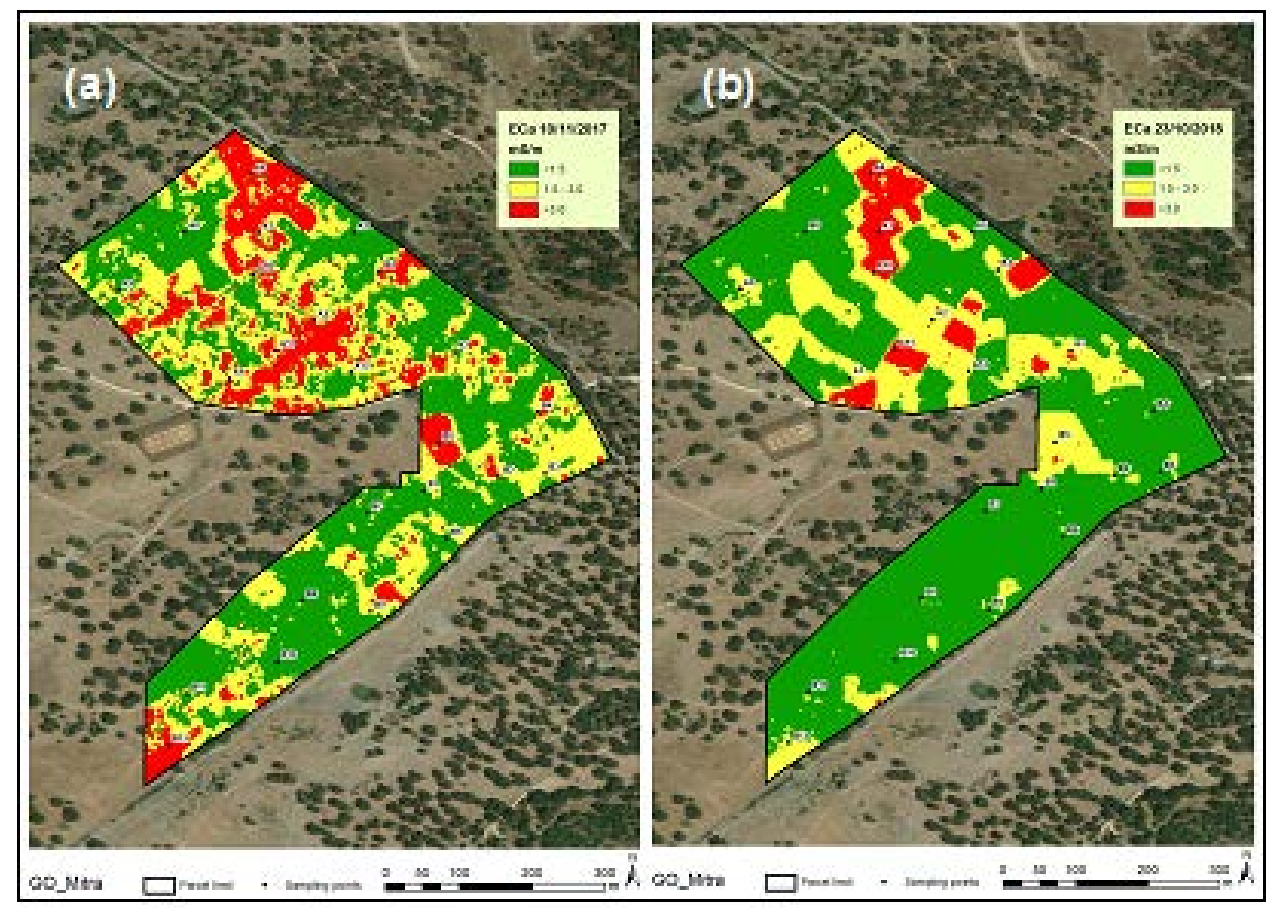

Figura 2. Mapas da CEa na camada superficial do solo $(0-0,30 \mathrm{~m})$ da parcela.

A Tabela 2 mostra, por um lado, que os valores médios da CEa na parcela experimental são relativamente baixos, o que reflecte a fraca textura do solo [6] e, por outro, uma acentuada variabilidade espacial da CEa (CV da ordem dos $50 \%$ ).

É possível verificar, de 2017 para 2018, uma ligeira diminuição dos valores médios da CEa na parcela experimental, facto que pode estar associado à diminuição dos teores médios de humidade do solo [19]. A Figura 3 ilustra a forte correlação $(R 2=0,71)$ verificada entre estas duas variáveis (humidade e CEa) na parcela experimental. A acentuada variabilidade espacial da CEa na parcela experimental mostra o interesse potencial desta técnica e da informação resultante para efeitos de apoio à tomada de decisão em termos de definição de ZHG. 
Tabela 2. Condutividade eléctrica aparente e humidade do solo na camada superficial (0-0,30 m) da parcela experimental.

\begin{tabular}{lccc}
\hline Parâmetros do solo & Média $\pm \mathrm{DP}$ & $\mathbf{C V}, \%$ & Intervalo de variação \\
\hline $\mathrm{CE}$ a, $\mathrm{mS} / \mathrm{m}$ & & & \\
$10 / 11 / 2017$ & $2,3 \pm 1,3$ & 56,5 & {$[0,8-5,5]$} \\
$23 / 10 / 2018$ & $1,8 \pm 0,9$ & 50,0 & {$[0,6-3,7]$} \\
\hline Humidade, \% & & & \\
$10 / 11 / 2017$ & $9,4 \pm 1,7$ & 19,1 & {$[7,4-12,5]$} \\
$23 / 10 / 2018$ & $7,9 \pm 1,0$ & 12,7 & {$[6,4-9,8]$} \\
\hline
\end{tabular}

DP- Desvio padrão; CV- Coeficiente de variação; CEa- Condutividade eléctrica aparente do solo;

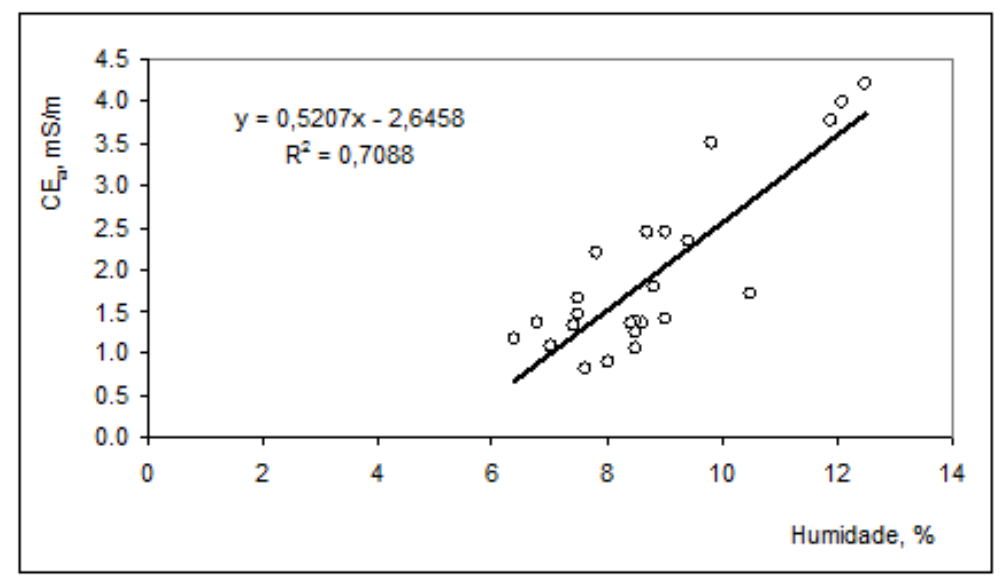

Figura 3. Relação entre a humidade e a condutividade eléctrica aparente do solo $\left(\mathrm{CE}_{\mathrm{a}}\right)$ na camada superficial $(0-0,30 \mathrm{~m})$ da parcela experimental.

A Figura 4 ilustra os mapas do NDVI (a) e do NDWI (b) médios na parcela experimental obtidos a partir das imagens de satélite (Sentinel-2) em séries temporais ao longo do Inverno e da Primavera de 2017 e de 2018. A Tabela 3 mostra que a variabilidade do solo (expressa pelos mapas da $\mathrm{CEa}$ ) não se reflecte na variabilidade espacial do desenvolvimento da pastagem (CV da ordem dos 4 a $8 \%$, respectivamente para o NDVI e para o NDWI) no período de maior vigor vegetativo (Outono e Primavera).

A Figura 5 mostra uma forte correlação $\left(\mathrm{R}^{2}=0,75\right)$ entre os dois índices (NDVI e NDWI), o que demonstra que ambos podem ser utilizados para monitorizar a evolução do estado desenvolvimento da pastagem, uma vez que o primeiro reflecte fundamentalmente o teor de clorofila e o segundo o teor de água das plantas, ambos indicadores do vigor vegetativo da pastagem [19].

A Figura 6 ilustra os mapas de classes de gestão da CEa do solo (a), do NDVI (b) e do NDWI (c) da parcela experimental e ainda o mapa do potencial produtivo acumulado. O mapa de classes de gestão resultante da análise da variabilidade espacial e da estabilidade temporal da CEa do solo (Figura 6 (a)) mostra instabilidade numa área significativa da parcela, o que pode dificultar a tomada de decisão quanto à definição das ZHG. O padrão da humidade do solo, que sofre influência do relevo do terreno, pode assim perturbar e sobrepor-se a outras variáveis associadas às características do solo (nomeadamente os teores de argila, a CTC, o pH ou os teores de matéria orgânica). 


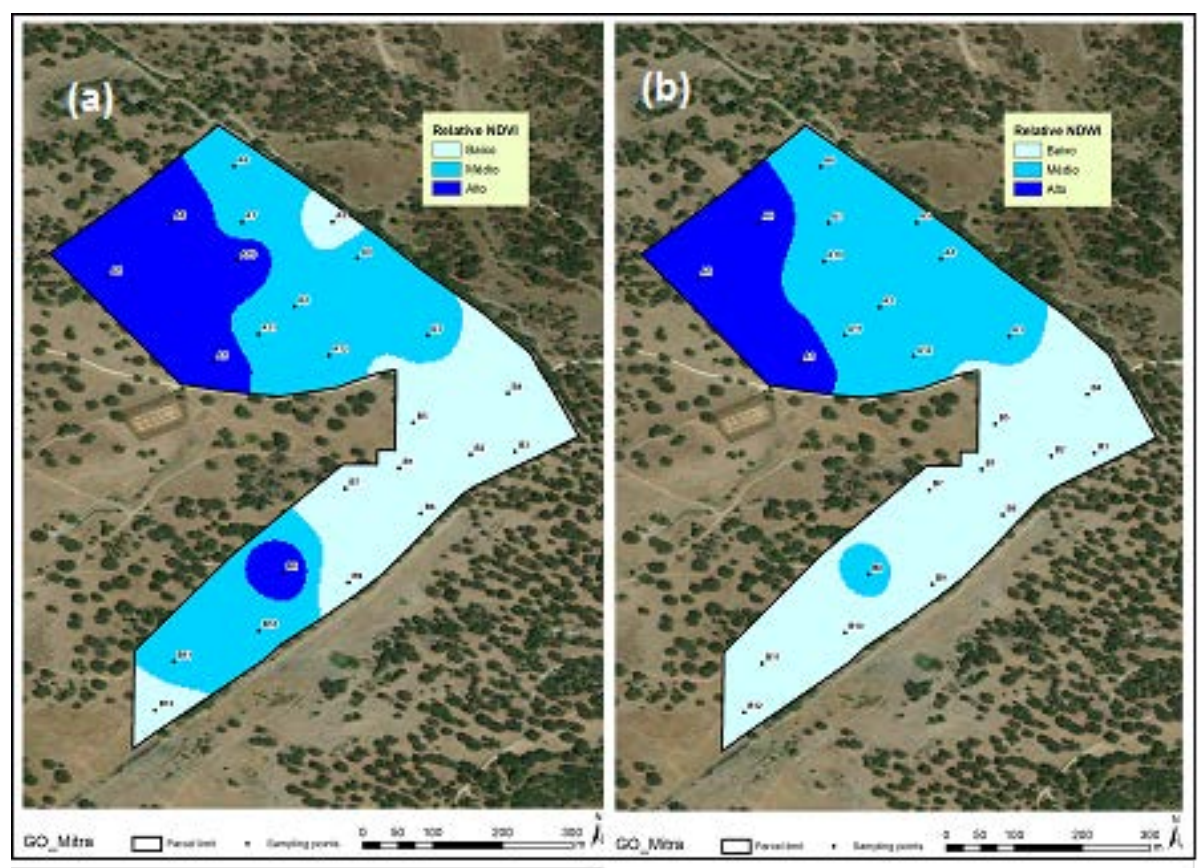

Figura 4. Mapas do NDVI (a) e do NDWI (b) médios da parcela experimental obtidos a partir das imagens de satélite (Sentinel-2) em séries temporais ao longo do Inverno e da Primavera de 2017 e de 2018.

Tabela 3. NDVI e NDWI da parcela experimental obtidos a partir das imagens de satélite (Sentinel-2) em séries temporais ao longo do Inverno e Primavera de 2017 e de 2018.

\begin{tabular}{cccc}
\hline Índices & Média \pm DP & $\mathbf{C V}, \%$ & Intervalo de variação \\
\hline NDVI & $0,630 \pm 0,024$ & 3,8 & {$[0,588-0,684]$} \\
NDWI & $0302 \pm 0,025$ & 8,3 & {$[0,235-0,352]$} \\
\hline
\end{tabular}

DP- Desvio padrão; CV- Coeficiente de variação; NDVI- "Normalized difference vegetation index" ; NDWI- "Normalized difference water index";

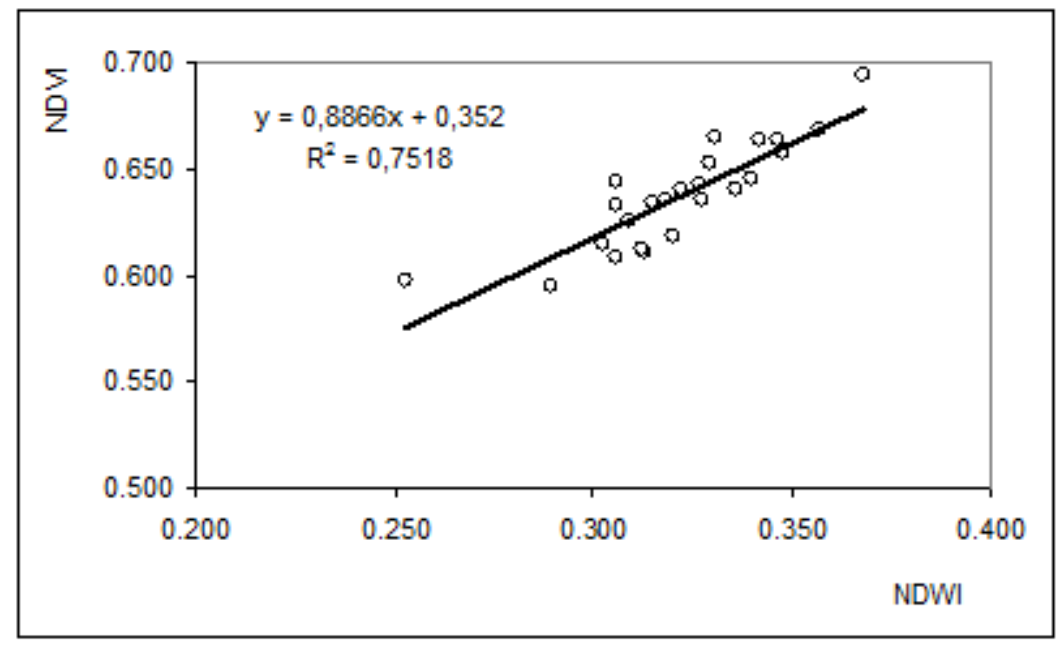

Figura 5. Relação entre os valores médios do NDWI e do NDVI obtidos nos períodos de Inverno e de Primavera de 2017 e 2018 a partir das imagens de satélite (“Sentinel-2") no conjunto dos pontos de amostragem da parcela experimental. 

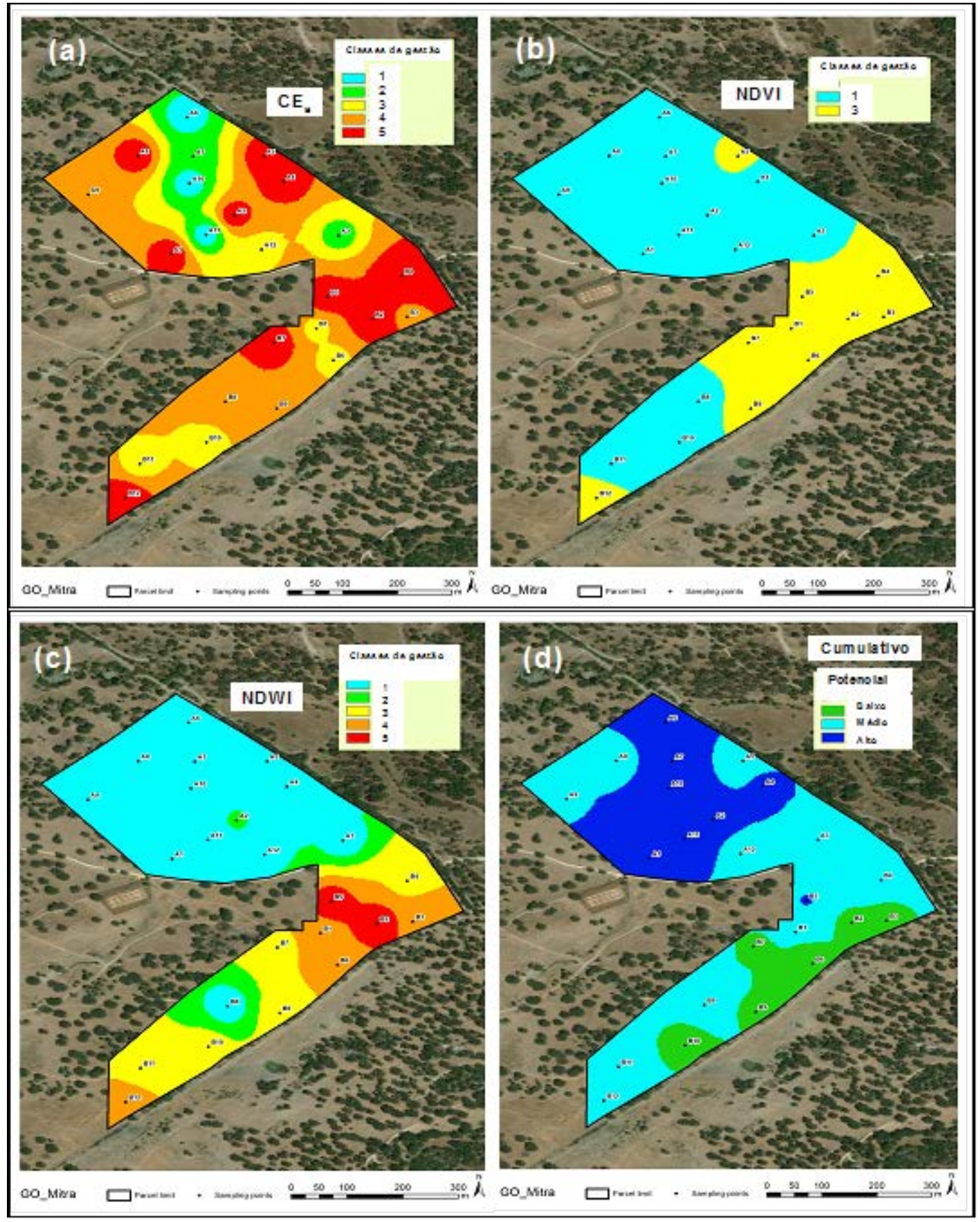

Figura 6. Mapas de classes de gestão da CEa do solo (a), do NDVI (b) e do NDWI (c) da parcela experimental e mapa do potencial produtivo acumulado (d).(Classe 1- superior à média e estável; Classe 2- superior à média e moderadamente estável; Classe 3- inferior à média e estável; Classe 4- Inferior à média e moderadamente estável; Classe 5- instável).

No caso concreto, mais do que definir padrões de variabilidade espacial, o levantamento da $\mathrm{CE}_{a}$ do solo permitiu identificar uma área de deficiente drenagem, a que correspoderam valores mais elevados da $\mathrm{CE}_{a}$ na zona central da parcela, num gradiente N-S (zonas de amostragem, 6, 7, 10 e 11). Será importante repetir o levantamento da $C E_{a}$ do solo nesta parcela experimental para perceber se os padrões de variabilidade espacial estabilizam. A área restante mostra uma tendência para valores mais elevados da CEa na zona mais a Norte, em contraste com a zona mais a Sul. Por outro lado, os mapas de classes de gestão do NDVI e do NDWI 


\section{CONGRESO IBÉRICO DE AGROINGENIERÍA \\ X CONGRESSO IBÉRICO DE AGROENGENHARIA \\ 3 - 6 septiembre 2019, Huesca - España}

mostram, por um lado, uma predominância clara das áreas estáveis ou moderadamente estáveis, relativamente às áreas instáveis e, por outro, confirmam o maior vigor vegetativo da pastagem na zona a Norte da parcela, indiciando uma boa relação com a $\mathrm{CE}_{a}$ do solo [6].

A integração em simultâneo das 3 variáveis consideradas (CEa, NDVI e NDWI) ilustra uma proposta de 3 ZHG (Figura 6, (d)) tendo por base o potencial produtivo de cada zona da parcela: zonas com potencial alto correspondem a valores relativamente elevados das 3 variáveis; zonas com potencial médio terão pelo menos uma das variáveis com valores relativamente elevados; zonas de potencial baixo apresentam todas as variáveis com valores relativamente baixos.

Estes resultados mostram o interesse em integrar a informação da $\mathrm{CE}$ a do solo com os índices obtidos a partir das imagens de satélite para identificar e validar ZHG para apoio à tomada de decisão na gestão diferenciada da fertilização. A decisão final do gestor deverá ser no sentido de aplicar maiores quantidades de adubo nas zonas com maior potencial produtivo.

\section{Conclusões}

A aplicação racional de fertilizantes em pastagens utilizando tecnologia VRT pressupõe, numa fase prévia, a identificação de "zonas homogéneas de gestão" (ZHG). Neste trabalho a avaliação da variabilidade espacial e da estabilidade temporal da condutividade eléctrica aparente do solo e da resposta da cultura (através dos índices NDVI e NDWI obtidos a partir das imagens de satélite) em dois anos consecutivos (2017 e 2018) permitiram primeiro identificar e depois validar a definição de ZHG. Estas ZHG serão a base para a recolha inteligente de amostras de solo e de pastagem de forma a quantificar as necessidades de fertilização diferenciada, constituindo assim o mapa final de prescrição.

\section{Agradecimentos}

Este trabalho foi suportado através do "Programa 1.0.1.-Grupos Operacionais" no âmbito dos projectos "PDR2020-101-030693" e "PDR2020-101-031244" e através da FCT (Fundação para a Ciência e Tecnologia) no âmbito do projecto “UID/AGR/00115/2019”.

\section{Referências}

1. Serrano J., Shahidian S., Marques da Silva J. Gestão diferenciada das pastagens baseada na monitorização da condutividade eléctrica aparente do solo. Revista de Ciências Agrárias. 2018, 41(4), 881-892.

2. Efe Serrano J. Pastures in Alentejo: technical basis for characterization, grazing and improvement. Universidade de Évora - ICAM (Ed.), Évora, Portugal: Gráfica Eborense. 2006, 165-178.

3. Serrano J., Peça J., Marques da Silva J., Shahidian S. Avaliação de tecnologias para aplicação diferenciada de fertilizantes: novos conceitos de gestão em pastagens permanentes. Revista de Ciências Agrárias. 2014, 37(3), 253-269.

4. Moral F., Terrón J., Marques da Silva J. Delineation of management zones using mobile measurements of soil apparent electrical conductivity and multivariate geostatistical techniques. Soil \& Tillage Research. 2010, 106, 335-343.

5. Peralta N. R., Costa J. L. Delineation of management zones with soil apparent electrical conductivity to improve nutrient management. Computers and Electronics in Agriculture. 2013, 99, 218-226.

6. Serrano J., Peça J., Marques da Silva J., Shahidian S. Mapping soil and pasture variability with an electromagnetic induction sensor. Computers and Electronics in Agriculture. 2010, 73, 7-16.

7. Lumbierres M., Méndez P. F., Bustamante J., Soriguer R., Santamaria L. Modeling biomass production in seasonal wetlands using Modis NDVI land surface phenology. Remote Sensing. 2017, $9,392$. 


\section{CONGRESO IBÉRICO DE AGROINGENIERÍA \\ X CONGRESSO IBÉRICO DE AGROENGENHARIA \\ 3- 6 septiembre 2019, Huesca - España}

8. Louhaichi M., Hassan S., Clifton K., Johnson D. E. A reliable and non-destructive method for estimating forage shrub cover and biomass in arid environments using digital vegetation charting technique. Agroforestry Systems. 2018, 92, 1341-1352.

9. Gu Y., Hunt E., Wardlow B., Basara J. B., Brown J. F., Verdin J. P. Evaluation of MODIS NDVI and NDWI for vegetation drought monitoring using Oklahoma Mesonet soil moisture data. Geophysical Research Letters. 2008, 35, L22401.

10. Serrano J., Shahidian S., Marques da Silva J. Monitoring seasonal pasture quality degradation in the Mediterranean montado ecosystem: proximal versus remote sensing. Water. 2018, 10(10), 1422.

11. Jackson T. J., Chen D., Cosh M., Li F., Anderson M., Walthall C., Doriaswamy P., Hunt E. R. Vegetation water content mapping using Landsat data derived normalized difference water index for corn and soybeans. Remote Sensing of Environment. 2004, 92, 475-482.

12. Serrano J., Shahidian S., Marques da Silva J. Evaluation of normalized difference water index as a tool for monitoring pasture seasonal and inter-annual variability in a Mediterranean agro-silvo-pastoral system. Water. 2019, 11(1), 62.

13. Sánchez-Ruiz S., Piles M., Sánchez N., Martínez-Fernández J., Vall-llossera M., Camps A. Combining SMOS with visible and near/shortwave/thermal infrared satellite data for high resolution soil moisture estimates. Journal of Hydrology. 2014, 516, 273-283.

14. FAO. World reference base for soil resources. Food and Agriculture Organization of the United Nations, World Soil Resources Reports № 103. 2006, Rome, Italy.

15. Blackmore, S. The interpretation of trends from multiple yield maps. Computers and Electronics in Agriculture. 2000, 26, 37-51.

16. Xu H.-W., Wang K., Bailey J., Jordan C., Withers A. Temporal stability of sward dry matter and nitrogen yield patterns in a temperate grassland. Pedosphere. 2006, 16, 735-744.

17. Serrano J., Peça J., Marques da Silva J., Shahidian S. Spatial and temporal stability of soil phosphate concentration and pasture dry matter yield. Precision Agriculture. 2011, 12, 214-232.

18. ESRI (Environmental Systems Research Institute) Inc. ArcView 9. 3 GIS Geostatistical Analyst. ESRI, Redlands, CA, USA. 2009.

19. Serrano J., Shahidian S., Marques da Silva J. Apparent electrical conductivity in dry versus wet soil conditions in a shallow soil. Precision Agriculture. 2013, 14, 99-114. 